\title{
Luteolin induces apoptosis in vitro through suppressing the MAPK and PI3K signaling pathways in gastric cancer
}

\author{
XUEYING LU ${ }^{1,2 *}$, YANHONG LI ${ }^{3 *}$, XIAOBO LI ${ }^{1,2}$ and HAJI AKBER AISA ${ }^{1,2}$ \\ ${ }^{1}$ The Key Laboratory of Plant Resources and Chemistry of Arid Zone, Xinjiang Technical Institute of Physics \\ and Chemistry, Chinese Academy of Sciences; ${ }^{2}$ State Key Laboratory Basis of Xinjiang Indigenous Medicinal \\ Plants Resource Utilization, Urumqi, Xinjiang 830011; ${ }^{3}$ Xinjiang Key Laboratory of Special Species Conservation \\ and Regulatory Biology, College of Life Science, Xinjiang Normal University, Urumqi, Xinjiang 830054, P.R. China
}

Received September 30, 2015; Accepted March 10, 2017

DOI: $10.3892 / \mathrm{ol} .2017 .6380$

\begin{abstract}
Luteolin, an active component of traditional Chinese medicine, exhibits potential for anti-tumor proliferation; however, the molecular events occurring in such process and the signal transduction pathways involved are currently unknown. Our group previously reported that luteolin inhibited proliferation and induced apoptosis in the gastric cancer cell line BGC-823. The aim of the present study was to investigate the mechanism by which the mitogen-activated protein kinase (MAPK) and phosphatidylinositol-4,5-bisphosphate 3-kinase (PI3K) signaling pathways regulate the apoptosis in vitro of BGC-823 cells following treatment with luteolin. It was observed that luteolin induced apoptosis through the intrinsic pathway by increasing the levels of caspase-3, caspase- 9 and cytochrome $c$, and the ratio of B-cell lymphoma (Bcl)-2 associated X protein (Bax) to Bcl-2. Luteolin suppressed the phosphorylation of extracellular signal-regulated kinase in the MAPK signaling pathway, as well as suppressing the phosphorylation of AKT, PI3K and mechanistic target of rapamycin in the PI3K signaling pathway. In addition, luteolin combined with LY294002 markedly increased the Bax/Bcl-2 ratio, while when combined with U0126, luteolin had less effects on the $\mathrm{Bax} / \mathrm{Bcl}-2$ ratio compared with luteolin treatment alone, suggesting that both the MAPK and PI3K signaling pathways are involved in the apoptosis induced by luteolin. Furthermore, luteolin attenuated the MAPK and PI3K signaling pathways
\end{abstract}

Correspondence to: Dr Xiaobo Li or Dr Haji Akber Aisa, The Key Laboratory of Plant Resources and Chemistry of Arid Zone, Xinjiang Technical Institute of Physics and Chemistry, Chinese Academy of Sciences, 40-1 Beijing Road, Urumqi, Xinjiang 830011, P.R. China

E-mail: 1_xb@outlook.com

E-mail: haji@ms.xjb.ac.cn

*Contributed equally

Key words: luteolin, gastric carcinoma, MAPK, PI3K, DUSPs, CXCL16 by increasing the expression of specific dual-specificity phosphatases and decreasing the expression of chemokine (C-X-C motif) ligand 16 at the messenger RNA level, respectively. Taken together, the present results demonstrate that luteolin is a potential chemotherapeutic agent against gastric cancer by exerting a dual inhibition on the MAPK and PI3K signaling pathways.

\section{Introduction}

Gastric carcinoma (GC) is the third most common cause of cancer mortality worldwide, and $>50 \%$ of GC cases occur in Eastern Asia (1,2). Therapy for GC includes surgical resection, radiation and chemotherapy (3). Surgical resection is the curative treatment for patients with early stages of disease. However, $20 \%$ of patients survive 5 years after surgery, and the majority of patients with advanced GC, which is characterized by poor prognosis and metastasis, eventually relapse (4). At present, the use of chemotherapy and combination treatments are alternative therapeutic strategies for controlling advanced GC (5). Therefore, it is an urgent requirement to identify new chemotherapeutic agents for preventing gastric cancer metastasis and improving the 5-year survival rates of gastric cancer patients $(6,7)$.

A malignant tumor could be developed from a normal cell in various mechanisms, including self-sufficiency in growth signals, insensitivity to antigrowth signals, evasion of apoptosis, limitless replicative potential, sustained angiogenesis, tissue invasion and metastasis (8). Regarding growth signals, the mitogen-activated protein kinase (MAPK) and phosphatidylinositol-4,5-bisphosphate 3-kinase (PI3K) signaling pathways serve crucial roles in controlling fundamental cellular processes, including growth, proliferation, differentiation, migration and apoptosis (9). Emerging evidence has suggested that sustained activation of the MAPK and PI3K signaling pathways is responsible for anti-apoptosis and carcinogenesis (10). Data from several groups have suggested that constitutively activated extracellular signal-regulated kinase (ERK) is involved in the progression of certain types of human cancer, including carcinomas of the breast (11), colon (12) and prostate (13). Activation of the PI3K signaling pathway was significantly associated with the tumor development and 
progression of human gastric cancer, based on 56 gastric cancer specimens (14). In addition, An et al (15) investigated a total of 290 patients with pT2b gastric cancer and elucidated that phosphorylated (p-) mammalian target of rapamycin (mTOR) was expressed in patient-derived gastric cancer samples, and that mTOR activation was associated with the extent of lymph node metastasis and poor survival in patients with gastric cancer. Therefore, inhibition of the PI3K and MAPK signal transduction pathways may represent a promising strategy in the treatment of the initiation and progression of gastric cancer.

A body of studies suggest that luteolin (3',4',5,7-tetrahydroxyflavone), a natural flavonoid compound highly enriched in a number of medicinal herbals, including Lonicera japonica, Scutellaria barbata and Ajuga decumbus (16), possesses diverse biological activities, including anti-inflammatory (17), antioxidant (18) and antiproliferative effects (19). Furthermore, it has been documented that luteolin could arrest the cell cycle and induce apoptosis in a wide variety of cancer cells in vitro, including prostate cancer cells (20), AGS human gastric cancer cells $(21,22)$, SMMC7721 liver cancer cells (23), COLO205 human colorectal cancer cells and HeLa human cervical cancer cells (24). It has also been reported that luteolin was able to significantly decrease colon cancer incidence and the number of tumors per rat when administered at the initiation and post-initiation stages of carcinogenesis (25). To date, various well-controlled clinical trials have been carried out to evaluate the chemopreventive potential of luteolin in human subjects (26-30). However, the molecular events and signal transduction pathways involved in the mechanism of action of luteolin in gastric cancer remain to be elucidated.

Therefore, the purpose of the present study was to investigate the role of the MAPK and PI3K signaling pathways in regulating luteolin-induced apoptosis in vitro. The present findings highlight the potential of luteolin as an anti-cancer therapeutic agent that targets MAPK and PI3K signaling in gastric cancer cells.

\section{Materials and methods}

Reagents and antibodies. Luteolin, MTT and dimethyl sulfoxide were purchased from Sigma-Aldrich (Merck KGaA, Darmstadt, Germany). RPMI-1640 medium, fetal bovine serum (FBS), penicillin and streptomycin were purchased from Gibco (Thermo Fisher Scientific, Inc. Waltham, MA, USA). Annexin V-FITC apoptosis detection kit and anti-cytochrome $c$ antibody (catalogue no. 556433) were purchased from BD Biosciences (San Jose, CA, USA). Primary antibodies, including anti-p-PI3K (Y607; catalogue no. YP0765), anti-p-mTOR (S2448; catalogue no. YP0176) and anti- $\beta$-actin (catalogue no. YT0099) antibodies, were from ImmunoWay Biotechnology Company (Plano, TX, USA), while anti-p-AKT (Ser473; catalogue no. 4051), antip-p38 (Thr180/Tyr182; catalogue no. 9216), anti-p-ERK1/2 (Thr202/Tyr204; catalogue no. 9106), anti-p-c-Jun N-terminal kinase (JNK) (Thr183/Tyr185; catalogue no. 4668), anti-B-cell lymphoma (Bcl)-2 (catalogue no. 15071), anti-Bcl-2 associated $\mathrm{X}$ protein (Bax) (catalogue no. 2772), anti-caspase-3 (catalogue no. 9668) and anti-caspase-9 (catalogue no. 9508) antibodies were purchased from Cell Signaling Technology, Inc. (Danvers, MA, USA). Caspase-3 (3G2, catalogue no. 9668) is mouse monoclonal antibody that detects endogenous levels of full length $(35 \mathrm{kDa})$ and the large fragment $(17 / 19 \mathrm{kDa})$ of caspase-3 resulting from cleavage at aspartic acid 175. Caspase-9 (C9, catalogue no. 9508) is mouse monoclonal antibody that detects endogenous levels of the pro form and cleaved fragments of caspase-9 (47,37 and $35 \mathrm{kDa})$. The horseradish peroxidase-conjugated secondary antibodies anti-rabbit immunoglobulin (Ig)G (catalogue no. sc-2357) and anti-mouse IgG (catalogue no. sc-516102) were purchased from Santa Cruz Biotechnology, Inc. (Dallas, TX, USA). U0126 (catalogue no. S1102) and LY294002 (catalogue no. S1105) were purchased from Selleck Chemicals (Houston, TX, USA). All other chemicals were purchased from Sangon Biology Engineering Technology Service, Ltd. (Shanghai, China) and were of analytical grade.

Cancer cell culture. The human gastric cancer cell line BGC-823 was obtained from the Cell Center at Chinese Academy of Medical Sciences and Peking Union Medical College (Beijing, China). The cells were maintained in RPMI-1640 medium supplemented with 10\% FBS, $100 \mathrm{U} / \mathrm{ml}$ penicillin and $100 \mu \mathrm{g} / \mathrm{ml}$ streptomycin at $37^{\circ} \mathrm{C}$ in a $5 \% \mathrm{CO}_{2}$ incubator. The cells were sub-cultured every 2 or 3 days and routinely checked visually under an inverted microscope (Leica Microsystems GmbH, Wetzlar, Germany) for potential contamination. No contamination was identified.

Western blot analysis. Cells $\left(1.0 \times 10^{6}\right)$ were seeded in $10-\mathrm{cm}$ dishes. When cells were in logarithmic growth phase, they were treated with luteolin at the $0,20,40$ and $60 \mu \mathrm{M}$ for $48 \mathrm{~h}$. Subsequently, BGC-823 cells were washed twice with PBS (pH 7.4) and lysed in $100 \mu \mathrm{l}$ radioimmunoprecipitation assay buffer (AR0102) that purchased from Boster Biotech (Wuhan, China). The lysed cells were removed from the culture dish by gentle scraping with a cell scraper (\#3010; Corning Incorporated, USA) and transferred to a microcentrifuge tube. The samples were centrifuged at $13,000 \mathrm{rpm}$ for $5 \mathrm{~min}$ at $4^{\circ} \mathrm{C}$, and the supernatant was then transferred to a new tube. Total protein concentration was determined using the Pierce BCA Protein assay kit (Thermo Fisher Scientific, Inc.). Proteins were separated by $10 \%$ SDS-PAGE and electrotransferred to a polyvinylidene fluoride membrane $(0.2 \mu \mathrm{m}$; Merck KGaA, Darmstadt, Germany) using a Trans-Blot SD Semi-Dry Transfer Cell (Bio-Rad Laboratories, Inc., Hercules, CA, USA). Blocking was carried out for $2 \mathrm{~h}$ in TBST [Tris-buffered saline (TBS) containing 1\%o Tween-20, v/v] with 5\% non-fat milk at room temperature. The primary antibodies $(1: 1,000)$ were incubated with the membrane overnight at $4^{\circ} \mathrm{C}$. Following three washes in TBST, secondary antibodies $(1: 2,000)$ were added and incubated at room temperature for $2 \mathrm{~h}$. The blots were washed with TBST three times, and detection was then performed using Pierce ECL Western Blotting Substrate (Thermo Fisher Scientific, Inc.).

Reverse transcription-quantitative polymerase chain reaction (qPCR) assay. After BGC-823 cells were exposed to 0, 20, 40 and $60 \mu \mathrm{M}$ luteolin for $48 \mathrm{~h}$, total RNA was extracted using TRIzol reagent (Invitrogen; Thermo Fisher Scientific, Inc.) according to the manufacturer's protocol. RNA concentration and purity were determined based on the measurement of 
absorbance at 260 and $280 \mathrm{~nm}$. RNase-free DNase I (Takara Bio, Inc., Japan) was used to remove the DNA contamination. M-MLV Reverse Transcriptase (Fermentas, Thermo Fisher Scientific, Inc.; Pittsburgh, PA, USA) was used according to the manufacturer's protocol to treat $2 \mu \mathrm{g}$ total RNA for synthesizing first-strand complementary DNA (cDNA). The cDNA was then subjected to qPCR for evaluation of the relative messenger RNA (mRNA) levels. Gene-specific amplification was performed using an StepOne ${ }^{\mathrm{TM}}$ (96 wells) Real-Time PCR system (Applied Biosystems ${ }^{\circledR}$ ABI; Thermo Fisher Scientific, Inc.) with a $20 \mu \mathrm{l}$ PCR reaction mixture containing $1 \mu \mathrm{l}$ cDNA (synthesized as described above), $10 \mu \mathrm{l} 2 \mathrm{X}$ Fast SYBR-Green Master Mix (Applied Biosystems ${ }^{\circledR}$ ABI, USA), forward and reverse primers with a final concentration of $0.25 \mu \mathrm{M}$. The amplification conditions were $95^{\circ} \mathrm{C}$ for $15 \mathrm{~min}$, followed by 40 cycles of $95^{\circ} \mathrm{C}$ for $15 \mathrm{sec}, 56^{\circ} \mathrm{C}$ for $20 \mathrm{sec}$ and $72^{\circ} \mathrm{C}$ for $30 \mathrm{sec}$. The relative expression levels of the target genes were normalized to the geometric mean of the internal control gene, $G A P D H$. Each gene was performed in a set of three replicates. No template control was included in all batches. The $\mathrm{Cq}$ (threshold cycles) values were used to calculate the mRNA levels by the formula $2^{-\Delta \Delta C t}=2^{-[\Delta \mathrm{Ct} \text { treatment- } \Delta \mathrm{Ct} \text { control] }}$ (31). The primers used in qPCR analysis were obtained from Sangon Biology Engineering Technology Service, Ltd. (Shanghai, China), and their sequences are reported in Table I.

Statistical analysis. The data were expressed as the mean \pm standard deviation $(n=3)$. All calculations were performed with SPSS version 16 (SPSS, Inc., Chicago, IL, USA). Statistical significance was analyzed by one-way analysis of variance. $\mathrm{P}<0.05$ was considered to indicate a statistically significant difference.

\section{Results}

Luteolin induces activation of caspases. Our group previously reported that luteolin inhibited BGC-823 cell proliferation and induced apoptosis in a dose-dependent manner (32). In the present study, to further examine whether luteolin-induced apoptosis resulted from activating caspase enzymes, the activities of initiator caspase (caspase-9) and effector caspase (caspase-3) $(33,34)$ were measured by western blotting in BGC-823 cells. The results revealed that luteolin treatment increased the expression of cleaved caspase- 9 and caspase- 3 in a dose-dependent manner (Fig. 1). Next, the level of cytochrome $c$ in the cytoplasm was detected (35-38), as this is a key step in the process of caspase activation during apoptosis. As shown in Fig. 1, luteolin induced an increase of cytoplasmatic cytochrome $c$ in a dose-dependent manner. These results implied that luteolin-induced apoptosis in BGC-823 cells may occur through the intrinsic pathway.

Effects of luteolin on the expression of Bcl-2 family proteins. The involvement of the intrinsic pathway in apoptosis is regulated by proteins of the Bcl-2 family, which comprises anti-apoptotic (e.g., Bcl-2) and pro-apoptotic (e.g., Bax) proteins (39). The ratio of $\mathrm{Bax} / \mathrm{Bcl}-2$ could determine whether the cell undergoes apoptosis $(40,41)$. In the present study, luteolin treatment of $\mathrm{BGC}-823$ cells resulted in decreased of $\mathrm{Bcl}-2$ in a dose-dependent manner (Fig. 2A). Compared with that in
Table I. Primers used in quantitative polymerase chain reaction analysis.

\begin{tabular}{ll}
\hline Gene name & \multicolumn{1}{c}{ Primer sequence (5'-3') } \\
\hline FUSP1 & R: TTGAGGGTCACTACCAG \\
FUSP2 & F: AGTCACTCGTCAGACC \\
R: TGTTCTTCACCCAGTCAAT & F: CAAAGGCGGCTATGAG \\
RUSP4 & R: GGTTATCTTCCACTGGG \\
& F: CTGAGTGTTGCGTGGA \\
RUSP5 & FGTCTATTGCTTCTTGAAAGT \\
& R: CGAGACCCCAATAGTGC \\
FUSP6 & F: TCATTGACGAAGCCCG \\
& R: GCGTATTGAGTGGGAACA \\
FUSP7 & R: ATCCGCTACATCCTCAA \\
RUSP9 & F: CTGATCATAGGCATCGTT \\
& R: GGTGTAAGGATTCTCGGT \\
FUSP10 & F: CAGCAAGCCAAGAGGA \\
& R: TGACAAAGGCATAGAGCA \\
CXCL16 & F: AAGGTCGGAGTCAACGGATT \\
RAPDH & R: CTCCTGGAAGATGGTGATGG \\
&
\end{tabular}

DUSP, dual-specificity phosphatase; CXCL16, chemokine (C-X-C motif) ligand 16; F, forward; R, reverse.

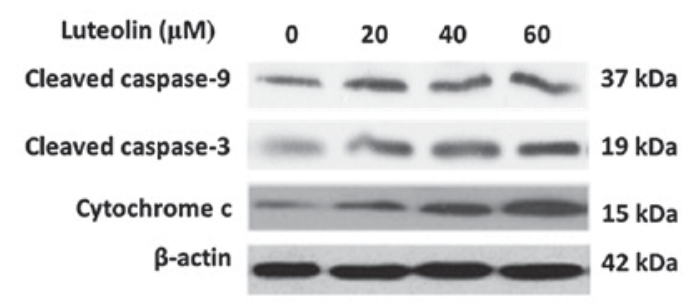

Figure 1. Luteolin induces the activation of caspases in BGC-823 cells. Cells were seeded in $60-\mathrm{mm}$ plates and cultured to $80-90 \%$ confluence. The cells were then treated with various doses of luteolin $(20,40$ and $60 \mu \mathrm{M})$ for $48 \mathrm{~h}$. Cells treated with dimethyl sulfoxide alone were used as the control. Wholecell extracts were subjected to immunoblot analysis using antibodies specific for caspase-9, caspase-3, cytochrome $c$ and $\beta$-actin.

control cells, the ratio of $\mathrm{Bax} / \mathrm{Bcl}-2$ significantly increased at the highest concentrations of luteolin in treated cells (Fig. 2B). These data further suggested that luteolin induced apoptosis via the intrinsic pathway in BGC-823 cells.

Luteolin suppresses the PI3K and MAPK signaling pathways. The key elements of the PI3K and MAPK signaling pathways were evaluated by western blotting in BGC-823 cells exposed to luteolin at $0,20,40$ and $60 \mu \mathrm{M}$ for $48 \mathrm{~h}$. As shown in Fig. 3A, luteolin reduced the expression level of p-PI3K, p-AKT and p-mTOR in a dose-dependent manner. These results suggested that luteolin treatment suppressed the PI3K signaling pathway.

With regards to the MAPK signaling pathway, after BGC-823 cells were treated with luteolin at 0, 20, 40 and 


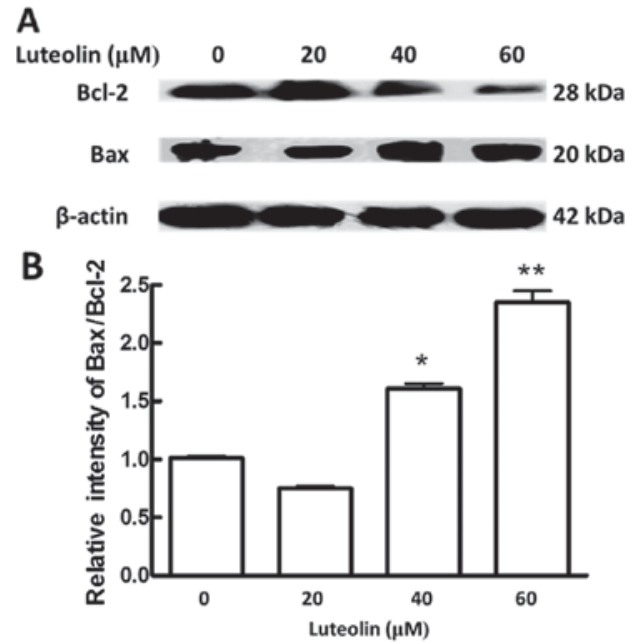

Figure 2. Effects of luteolin on the expression levels of Bcl-2 family proteins in BGC-823 cells. (A) Cells were treated as described above, and the cell extracts were subjected to immunoblotting using specific antibodies against $\mathrm{Bcl}-2$, Bax and $\beta$-actin. (B) The intensity of Bax/Bcl-2 protein signals was calculated. Values are the mean \pm standard deviation. The data were analyzed by one-way analysis of variance. ${ }^{*} \mathrm{P}<0.05$ and ${ }^{* *} \mathrm{P}<0.01$ compared with the control group. Bcl, B-cell lymphoma; Bax, Bcl-2 associated $\mathrm{X}$ protein.

$60 \mu \mathrm{M}$ for $48 \mathrm{~h}$, the results of western blotting indicated that the levels of p-ERK1/2 were reduced markedly in a dose-dependent manner, while the levels of p-p38 and p-JNK exhibited no significant change $(\mathrm{P}>0.05)$. These results suggested that luteolin treatment suppressed the ERK1/2 signaling pathway, but not the JNK or p38 signaling pathways, in BGC-823 cells (Fig. 3B).

To confirm the roles of the ERK1/2 or PI3K signaling pathways in luteolin-induced apoptosis in BGC-823 cells, the ERK inhibitor U0126 (42) and the AKT inhibitor LY294002 (43) were used for treating the cells in the absence or presence of $60 \mu \mathrm{M}$ luteolin. The effects were examined according to the ratio of Bax to $\mathrm{Bcl}-2$. The results indicated that exposure of BGC-823 cells to U0126 or LY294002 alone did not significantly alter the $\mathrm{Bax} / \mathrm{Bcl}-2$ ratio compared with that of control cells (Fig. 3C and D; $\mathrm{P}>0.05$ ). The $\mathrm{Bax} / \mathrm{Bcl}-2$ ratio in the combination U0126 plus luteolin group increased compared with that in the control group, while such ratio was lower than that observed upon luteolin treatment alone $(\mathrm{P}<0.05)$. The Bax/Bcl-2 ratio in the combination LY294002 plus luteolin group increased compared with that in the control group, being much higher than that observed following luteolin treatment alone $(\mathrm{P}<0.05)$. Taken together, these results indicated that the ERK and PI3K signaling pathways were involved in the apoptosis induced by luteolin in BGC-823 cells. Notably, the Bax/Bcl-2 ratio in the LY294002 plus luteolin group was markedly higher than that in the U0126 plus luteolin group ( 3.7 vs. 1.6), respectively, indicating that the PI3K signaling pathway has much stronger effects on luteolin-induced apoptosis than the MAPK signaling pathway.

Effects of luteolin on the expression of dual-specificity phosphatase (DUSP) and chemokine ( $C-X-C$ motif) ligand 16 (CXCL16) genes in BGC-823 cells. Dual-specificity phosphatases (DUSPs) are a heterogeneous group of protein phosphatases that have ability to dephosphorylate both tyrosine and serine/threonine residues (44) and serve a critical role in the inactivation of different isoforms of MAPK (45) DUSPs share common features, including a cluster of basic amino acids as part of the kinase interactive motif (KIM) on their amino terminus. The KIM confers substrate specificity and is the least homologous region demonstrating individual substrate preferences $(46,47)$. Therefore, the transcription level of DUSP1-DUSP10 was examined. The mRNA levels of DUSP1, 2, 4 and 5 were upregulated in luteolin-treated BGC-823 cells compared with those in controls cells, while the mRNA levels of DUSP6, 7,9 and 10 did not change during the treatment (Fig. 4). DUSP1, 2, 4 and 5 prefer to use the ERK as their substrates. This result was consistent with the decrease of p-ERK protein in the western blotting results of the present study, indicating that luteolin exhibits the potential to regulate the expression of specific DUSP genes, resulting in an attenuation of the MAPK signaling pathway.

Chalabi-Dchar et al (48) reported that blocking CXCL16 activity abrogated activation of the PI3K/AKT pathway, implying the CXCL16 axis may regulate the activity of PI3K pathway. Therefore, the expression of CXCL16 was monitored at an mRNA level. The results showed that the CXCL16 mRNA level was greatly downregulated in a dose manner after treatment with luteolin, suggesting that luteolin efficiently suppressed the mRNA level of CXCL16. Hence, the attenuation of PI3K pathway may be ascribed to the suppression of CXCL16 in BGC-823 cell lines following luteolin treatment.

\section{Discussion}

In the present study, the apoptotic process induced by luteolin in BGC-823 cells was associated with the activities of caspases and the expression of $\mathrm{Bcl}-2$ family proteins, which is in agreement with previous reports regarding the pro-apoptotic effects of luteolin on other cancer cells, including the gastric cancer cell line AGS $(21,22)$, human prostate cancer cells (49) and the human colon cancer cell line HT-29 (50). However, the underlying mechanism of luteolin-induced apoptosis is not well understood yet.

MAPKs have been linked to diverse cellular events, including proliferation, senescence, differentiation, migration and apoptosis (51). A well-characterized apoptotic signaling cascade is regulated by MAPKs, including JNK, ERK and p38 MAPK (51). The ERK1/2 signaling pathway primarily responds to growth and differentiation factors, and the p38 and JNK signaling pathways primarily responds to stress conditions $(52,53)$. The present study noticed that the phosphorylation of ERK1/2 decreased significantly following luteolin treatment, while that of p38 or JNK did not change in the course of luteolin treatment, suggesting that p38 and JNK are not involved in the regulation of apoptosis in the BGC-823 cell line. It has been reported that ERK1/2 participates in apoptotic signaling via post-translational regulation of the Bcl-2 family members, including Bcl-2 interacting mediator of cell death (Bim) (54). Phosphorylation of Bim by ERK1/2 results in a change in the $\mathrm{Bax} / \mathrm{Bcl}-2$ ratio, which determines whether cell apoptosis occurs (55). In the present study, although U0126 treatment alone did not induce apoptosis in BGC-823 cells, when combined with luteolin, it increased the 
A

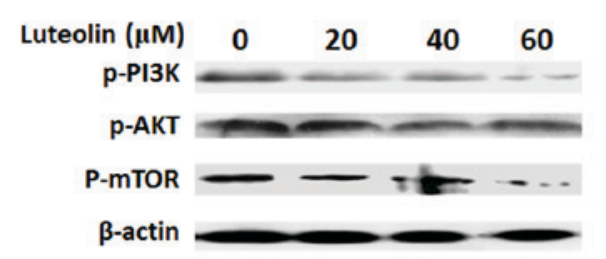

B

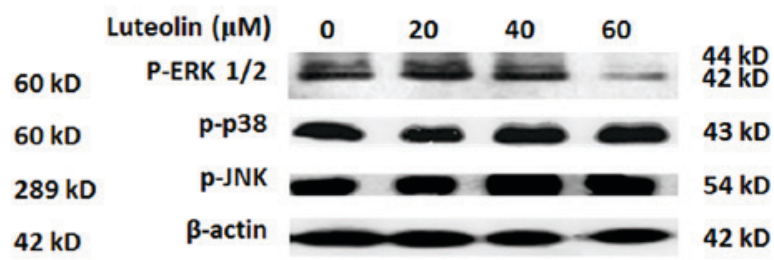

D

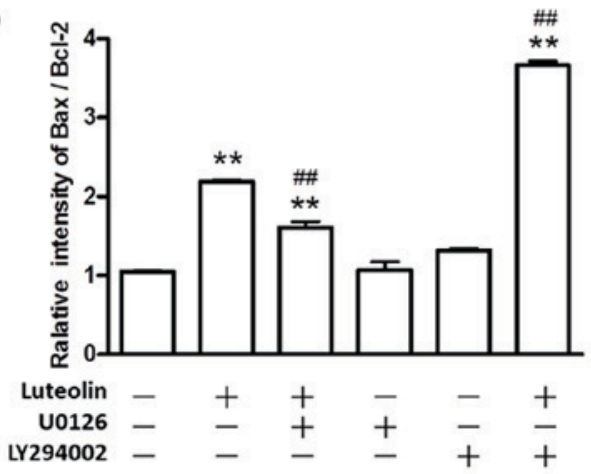

Figure 3. Luteolin inhibits the activation of the PI3K and MAPK signaling pathways in BGC-823 cells. Cells were seeded in 60 -mm plates and cultured to $80-90 \%$ confluence. The cells were then treated with various doses of luteolin $(20,40$ and $60 \mu \mathrm{M})$ for $48 \mathrm{~h}$. Cells treated with dimethyl sulfoxide alone were used as the control. (A) Luteolin inhibited the PI3K signaling pathway in BGC-823 cells. Cells were treated as described above, and the cell extracts were subjected to immunoblot analysis using anti-p-PI3K, anti-p-AKT, anti-p-mTOR and anti- $\beta$-actin antibodies. (B) Effect of luteolin on ERK1/2, p38 and JNK pathways. Cells were treated as described above, and the cell extracts were subjected to immunoblotting using anti-p-ERK, anti-p-p38, anti-p-JNK and anti$\beta$-actin antibodies. (C) Effects of luteolin on the Bcl-2 and Bax in BGC-823 cells. Cells were treated as described above, and the cell extracts were subjected to immunoblotting using anti-p-ERK, anti-Bcl-2, anti-Bax and anti- $\beta$-actin antibodies. (D) The relative intensity of Bax/Bcl-2. Values are the mean \pm standard deviation. The data were analyzed by one-way analysis of variance. ${ }^{* *}$ The control group vs. all the other groups $(\mathrm{P}<0.001)$; ${ }^{\# \#}$ luteolin treatment groups vs. luteolin+U0126 and luteolin+LY294002 treatment groups ( $\mathrm{P}<0.001)$. PI3K, phosphatidylinositol-4,5-bisphosphate 3-kinase; mTOR, mammalian target of rapamycin; p-, phosphorylated; ERK, extracellular signal-regulated kinase; JNK, c-Jun N-terminal kinase; Bcl, B-cell lymphoma; Bax, Bcl-2 associated X protein.

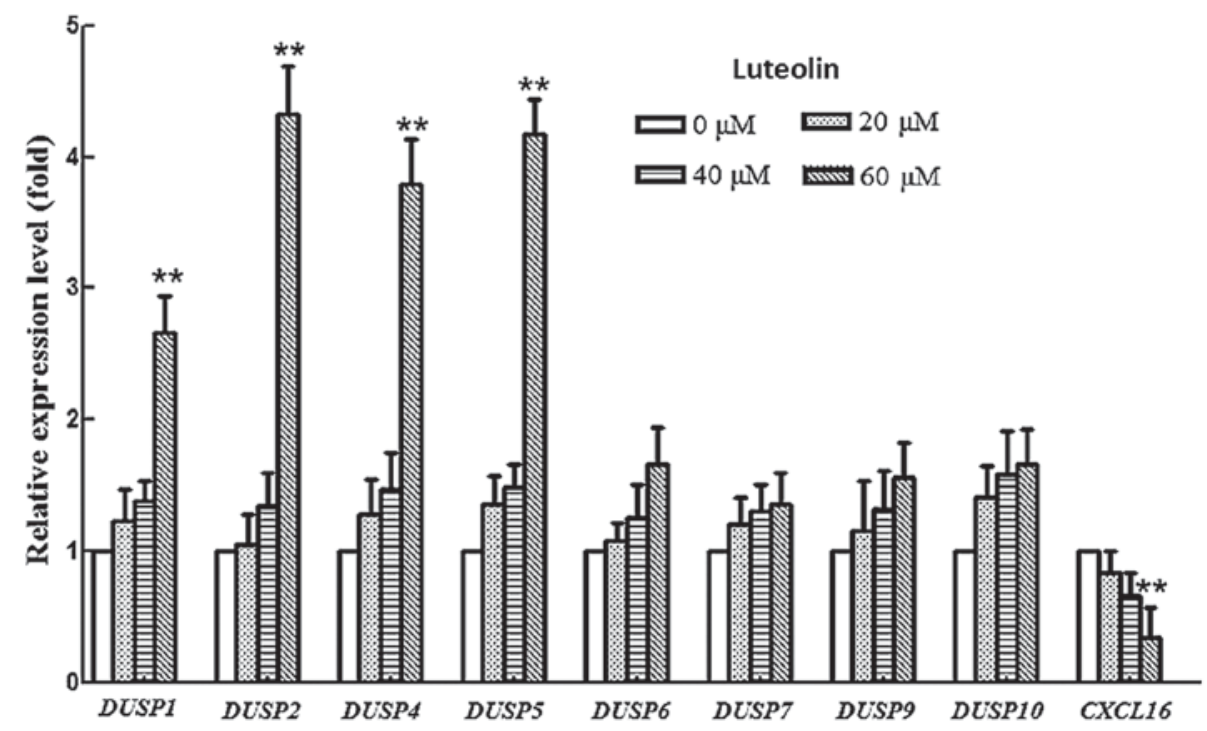

Figure 4. Effects of luteolin on the expression of DUSP and CXCL16 genes in BGC-823 cells. Values are the mean \pm standard deviation of three independent experiments. The data were analyzed by one-way analysis of variance. ${ }^{* *} \mathrm{P}<0.01$ compared with the control group. DUSP, dual-specificity phosphatase; CXCL16, chemokine (C-X-C motif) ligand 16.

$\mathrm{Bax} / \mathrm{Bcl}-2$ ratio, suggesting that luteolin-induced apoptosis was mediated by the ERK1/2 signaling pathway.

A major determinant of the biological outcome of MAPK signaling is the duration and magnitude of kinase activation, which can be achieved by serine/threonine phosphatases, tyrosine-specific phosphatases or dual-specificity phosphatases (DUSPs) $(56,57)$. DUSPs, whose family consists of 25 members, can specifically dephosphorylate $\geq 1$ MAPKs, and their substrate specificity is dependent on the cell type and context $(44,46)$. DUSP1, 2, 4 and 5 are mitogen- and 
stress-inducible nuclear DUSPs, which prefer using ERK as a substrate compared with JNK or p38. DUSP6, 7 and 9 are cytoplasmic ERK-specific DUSPs, while DUSP8 and 10 are JNK/p38-specific phosphatases present in both the nucleus and cytoplasm (58). The present study revealed that DUSP1, 2, 4 and 5 mRNA expression was upregulated, while the expression of other DUSPS was unchanged, upon luteolin treatment. These results indicated that highly expressed DUSP1,2, 4 and 5 specifically dephosphorylated ERK1/2 as a substrate, which may explain the decrease in phosphorylation of ERK1/2. No difference in p-JNK or p-p38 was observed between cells treated with or without luteolin, which may be due to the unchanged expression of other DUSPS. To date, no study has reported that luteolin has the potential to regulate the expression of DUSP genes on GC. Taken together, increased mRNA levels of specific DUSPS in luteolin-treated BGC-823 cell lines resulted in decreased p-ERK1/2 in the present study, and the suppression of p-ERK1/2 could increase the ratio of $\mathrm{Bax}$ to $\mathrm{Bcl}-2$, eventually triggering apoptosis.

The PI3K pathway relies on an array of intracellular events that have been intensively studied in previous years (59). The PI3K signaling pathway may influence apoptosis regulation, including the regulation of the Bcl-2 family proteins $(10,60)$. One of the important members of the Bcl-2 family of proteins is Bax, which can be phosphorylated at the inhibition site Ser184 near the C-terminus by AKT, leading to suppression of the apoptotic activity mediated by Bax (61). Thus, the activity of PI3K in cancer cells protects them from undergoing apoptosis; conversely, inhibiting the activity of PI3K can induce apoptosis in cancer cells $(62,63)$. In the present study, it was demonstrated that luteolin may inhibit p-PI3K, p-AKT and p-mTOR in the gastric cancer cell line BGC-823, suggesting that the PI3K signaling pathway may be involved in luteolin-induced apoptosis. Further experiments verified that combined treatment with LY294002 and luteolin had an enhanced effect on the induction of apoptosis, as evidenced by a significant increase in the $\mathrm{Bax} / \mathrm{Bcl}-2$ ratio, indicating that luteolin-induced apoptosis mainly occurred through the PI3K signaling pathway.

The activation of PI3K family members is a universal event in response to cytokines, growth factors and hormones $(59,64)$. Chemokines, a superfamily of chemotactic cytokines consisting of nearly 50 cytokine members and 20 chemokine receptors $(65,66)$, are classified into four major families based on the relative position of their cysteine residues near the $\mathrm{NH}_{2}$ terminus: CC, CXC, C and CX3C (67). Xing et al reported that aberrant expression of CXCL16 and CXCR6 may be involved in gastric carcinogenesis, and that the expression and serum concentration of CXCL16 could indicate the aggressiveness and prognosis of GCs (68). Furthermore, a previous study elucidated that PI3K/AKT/mTOR signaling may be involved in the CXCL16/CXCR6 biological axis (69). In that study, the phosphorylation of AKT was reduced with decreased CXCR6 expression, and mTOR was activated by CXCL16's stimulation of CXCR6 (69). Since the PI3K signaling pathway is involved in the activation of the CXCR6/CXCL16 axis (70,71), a number of therapy options may target blocking this axis or exploit other antibodies against this signaling pathway in order to prevent metastasis. Based on such strategy, various antibodies have been designed to specifically block the CXCL16/CXCR6 axis; however, the positive outcomes are poor (72-74). The present study demonstrated that luteolin efficiently inhibited CXCL16 mRNA expression. In the present study, based on the suppression of PI3K/AKT/mTOR signaling, it was hypothesized that luteolin may inhibit the expression of CXCL16, resulting in the suppression of the signaling PI3K pathway. To the best of our knowledge, the present study is the first to report that chemicals have the potential to regulate the CXCL16/CXCR6 axis, which may be beneficial in the development of a more effective anti-metastasis therapeutic strategy for gastric cancer. The present results also provide the first evidence suggesting that luteolin treatment may also alter the tumor microenvironment.

In summary, the present study has demonstrated that luteolin could induce apoptosis in the BGC-823 cell line through the intrinsic pathway. Luteolin upregulated the mRNA levels of specific DUSPs, which suppressed the protein phosphorylation of ERK1/2. In addition, luteolin attenuated the mRNA levels of CXCL16, leading to the suppression of the PI3K signaling pathway, and both the ERK1/2 and the PI3K signaling pathways were closely associated with the regulation of apoptosis in the gastric cancer cell line BGC-823. The present results provide useful information for considering luteolin as an attractive chemotherapeutic agent against gastric cancer.

\section{Acknowledgements}

The authors thank Professor Michael Guiver, National Research Council Canada, Ottawa (Ontario, Canada) for his critical review of the manuscript. The present study was supported by the Joint Funds of the National Natural Science Foundation of China (grant no. U1203203) and the West Light Foundation of the Chinese Academy of Sciences (grant no. 2016-QNXZ-B-5).

\section{References}

1. Ferlay J, Soerjomataram I, Ervik M, Dikshit R, Eser S, Mathers C, Rebelo M, Parkin DM, Forman D and Bray F: GLOBOCAN 2012 v1.0, Cancer Incidence and Mortality Worldwide: IARC CancerBase No. 11. International Agency for Research on Cancer, Lyon, France, 2013.

2. Rahman R, Asombang AW and Ibdah JA: Characteristics of gastric cancer in Asia. World J Gastroenterol 20: 4483-4490, 2014.

3. Menges M and Hoehler T: Current strategies in systemic treatment of gastric cancer and cancer of the gastroesophageal junction. J Cancer Res Clin Oncol 135: 29-38, 2009.

4. Green D, Ponce de Leon S, Leon-Rodriguez E and Sosa-Sanchez R: Adenocarcinoma of the stomach: Univariate and multivariate analysis of factors associated with survival. Am J Clin Oncol 25: 84-89, 2002

5. Ajani JA: Evolving chemotherapy for advanced gastric cancer. Oncologist 10 (Suppl 3): S49-S58, 2005.

6. Hejna M, Wöhrer S, Schmidinger M and Raderer M: Postoperative chemotherapy for gastric cancer. Oncologist 11: 136-145, 2006.

7. Di Costanzo F, Gasperoni S, Manzione L, Bisagni G, Labianca R, Bravi S, Cortesi E, Carlini P, Bracci R, Tomao S, et al: Adjuvant chemotherapy in completely resected gastric cancer: A randomized phase III trial conducted by GOIRC. J Natl Cancer Inst 100: 388-398, 2008

8. Yan Y, Wang LF and Wang RF: Role of cancer-associated fibroblasts in invasion and metastasis of gastric cancer. World $\mathbf{J}$ Gastroenterol 21: 9717-9726, 2015.

9. Mendoza MC, Er EE and Blenis J: The Ras-ERK and PI3K-mTOR pathways: Cross-talk and compensation. Trends Biochem Sci 36: 320-328, 2011. 
10. Trisciuoglio D, Iervolino A, Zupi G and Del Bufalo D: Involvement of PI3K and MAPK signaling in bcl-2-induced vascular endothelial growth factor expression in melanoma cells. Mol Biol Cell 16: 4153-4162, 2005.

11. Adeyinka A, Nui Y, Cherlet T, Snell L, Watson PH and Murphy LC: Activated mitogen- activated kinase expression during human breast tumorigenesis and breast cancer progression. Clin Cancer Res 8: 1747-1753, 2002.

12. Kress TR, Raabe T and Feller SM: High Erk activity suppresses expression of the cell cycle inhibitor p27Kip1 in colorectal cancer cells. Cell Commun Signal 8: 1, 2010.

13. Uzgare AR, Kaplan PJ and Greenberg NM: Differential expression and/or activation of P38MAPK, ERK1/2, and JNK during the initiation and progression of prostate cancer. Prostate 55: 128-139, 2003.

14. Lin HL, Yang MH, Wu CW, Chen PM, Yang YP, Chu YR Kao CL, Ku HH, Lo JF, Liou JP, et al: 2-Methoxyestradiol attenuates phosphatidylinositol 3-kinase/Akt pathway- mediated metastasis of gastric cancer. Int J Cancer 121: 2547-2555, 2007.

15. An JY, Kim KM, Choi MG, Noh JH, Sohn TS, Bae JM and Kim S: Prognostic role of p-mTOR expression in cancer tissues and metastatic lymph nodes in pT2b gastric cancer. Int J Cancer 126: 2904-2913, 2010.

16. Miean KH and Mohamed S: Flavonoid (myricetin, quercetin, kaempferol, luteolin, and apigenin) content of edible tropical plants. J Agric Food Chem 49: 3106-3112, 2001.

17. Nishitani Y, Yamamoto K, Yoshida M, Azuma T, Kanazawa K, Hashimoto $\mathrm{T}$ and Mizuno M: Intestinal anti-inflammatory activity of luteolin: Role of the aglycone in NF- $\kappa \mathrm{B}$ inactivation in macrophages co-cultured with intestinal epithelial cells. Biofactors 39: 522-533, 2013

18. Ashokkumar P and Sudhandiran P: Protective role of luteolin on the status of lipid peroxidation and antioxidant defense against azoxymethane-induced experimental colon carcinogenesis. Biomed Pharmacother 62: 590-597, 2008.

19. Ashokkumar P and Sudhandiran G: Luteolin inhibits cell proliferation during Azoxymethane-induced experimental colon carcinogenesis via Wnt/ $\beta$-catenin pathway. Invest New Drugs 29 : 273-284, 2011

20. Sakurai MA, Ozaki Y, Okuzaki D, Naito Y, Sasakura T, Okamoto A, Tabara $\mathrm{H}$, Inoue $\mathrm{T}$, Hagiyama $\mathrm{M}$, Ito $\mathrm{A}$, et al: Gefitinib and luteolin cause growth arrest of human prostate cancer PC-3 cells via inhibition of cyclin G-associated kinase and induction of miR-630. PLoS One 9: e100124, 2014.

21. Wu B, Zhang Q, Shen WM and Zhu J: Anti-proliferative and chemosensitizing effects of luteolin on human gastric cancer AGS cell line. Mol Cell Biochem 313: 125-132, 2008.

22. Wang HY, Quan K, Jiang YL, Wu JG and Tang XW: Effect of Luteolin and its combination with chemotherapeutic drugs on cytotoxicity of cancer cells. Zhejiang Da Xue Xue Bao Yi Xue Ban 39: 30-36, 2010 (In Chinese).

23. Ding S, Hu A, Hu Y, Ma J, Weng P and Dai J: Anti-hepatoma cells function of luteolin through inducing apoptosis and cell cycle arrest. Tumour Biol 35: 3053-3060, 2014.

24. Shi RX, Ong CN and Shen HM: Luteolin sensitizes tumor necrosis factor-alpha-induced apoptosis in human tumor cells. Oncogene 23: 7712-7721, 2004.

25. Manju V and Nalini N: Protective role of luteolin in 1,2-dimethylhydrazine induced experimental colon carcinogenesis. Cell Biochem Funct 25: 189-194, 2007.

26. Samy RP, Gopalakrishnakone P and Ignacimuthu S: Anti-tumor promoting potential of luteolin against 7,12-dimethylbenz(a) anthracene-induced mammary tumors in rats. Chem Biol Interact 164: 1-14, 2006.

27. Amin AR, Kucuk O, Khuri FR and Shin DM: Perspectives for cancer prevention with natural compounds. J Clin Oncol 27 2712-2725, 2009.

28. Pandurangan AK, Dharmalingam P, Ananda Sadagopan SK and Ganapasam S: Effect of luteolin on the levels of glycoproteins during azoxymethane-induced colon carcinogenesis in mice. Asian Pac J Cancer Prev 13: 1569-1573, 2012.

29. Sagawa H, Naiki-Ito A, Kato H, Naiki T, Yamashita Y, Suzuki S, Sato S, Shiomi K, Kato A, Kuno T, et al: Connexin 32 and luteolin play protective roles in non-alcoholic steatohepatitis development and its related hepatocarcinogenesis in rats. Carcinogenesis 36 $1539-1549,2015$

30. Kasala ER, Bodduluru LN, Barua CC and Gogoi R: Antioxidant and antitumor efficacy of Luteolin, a dietary flavone on benzo(a) pyrene-induced experimental lung carcinogenesis. Biomed Pharmacother 82: 568-577, 2016.
31. Schmittgen TD and Livak KJ: Analyzing real-time PCR data by the comparative C(T) method. Nat Protoc 3: 1101-1108, 2008

32. Lu Xueying, Li Yanhong, Xiao Xiangwen, Haji Akber Aisa and Li Xiaobo: Study on inhibition of luteolin on proliferation of human gastric cancer cell line BGC-823. Mod J Integr Tradit Chinese Western Med 3: 246-249, 2012.

33. Hengartner MO: The biochemistry of apoptosis. Nature 407: 770-776, 2000.

34. Thornberry NA and Lazebnik Y: Caspases: Enemies within. Science 281: 1312-1316, 1998.

35. Pradelli LA, Bénéteau M and Ricci JE: Mitochondrial control of caspase-dependent and -independent cell death. Cell Mol Life Sci 67: 1589-1597, 2010.

36. Otera $\mathrm{H}$ and Mihara K: Mitochondrial dynamics: Functional link with apoptosis. Int J Cell Biol 2012: 821676, 2012.

37. Kumar A, Ganini D and Mason RP: Role of cytochrome $\mathrm{c}$ in $\alpha$-synuclein radical formation: Implications of $\alpha$-synuclein in neuronal death in Maneb- and paraquat-induced model of Parkinson's disease. Mol Neurodegener 11: 70, 2016.

38. Lopez-Cruzan M, Sharma R, Tiwari M, Karbach S, Holstein D, Martin CR, Lechleiter JD and Herman B: Caspase-2 resides in the mitochondria and mediates apoptosis directly from the mitochondrial compartment. Cell Death Discov 2: pii: 16005 , 2016.

39. Rong $\mathrm{Y}$ and Distelhorst $\mathrm{CW}$ : Bcl-2 protein family members: Versatile regulators of calcium signaling in cell survival and apoptosis. Annu Rev Physiol 70: 73-91, 2008.

40. Li C, Wu X, Sun R, Zhao P, Liu F and Zhang C: Croton tiglium extract induces apoptosis via Bax/Bcl-2 pathways in human lung cancer A549 cells. Asian Pac J Cancer Prev 17: 4893-4898, 2016.

41. Zhang S, Qin F, Yang L, Xian J, Zou Q, Jin H, Wang L and Zhang L: Nucleophosmin mutations induce chemosensitivity in THP-1 leukemia cells by suppressing NF- $\mathrm{BB}$ Activity and regulating Bax/Bcl-2 expression. J Cancer 7: 2270-2279, 2016

42. Csibi A, Fendt SM, Li C, Poulogiannis G, Choo AY, Chapski DJ, Jeong SM, Dempsey JM, Parkhitko A, Morrison T, et al: The mTORC1 pathway stimulates glutamine metabolism and cell proliferation by repressing SIRT4. Cell 153: 840-854, 2013.

43. Sapey E, Greenwood H, Walton G, Mann E, Love A, Aaronson N, Insall RH, Stockley RA and Lord JM: Phosphoinositide 3-kinase inhibition restores neutrophil accuracy in the elderly: Toward targeted treatments for immunosenescence. Blood 123: 239-248, 2014.

44. Patterson KI, Brummer T, O'Brien PM and Daly RJ: Dual-specificity phosphatases: Critical regulators with diverse cellular targets. Biochem J 418: 475-489, 2009.

45. Theodosiou A and Ashworth A. MAP kinase phosphatases. Genome Biol 3: REVIEWS3009, 2002.

46. Camps M, Nichols A and Arkinstall S: Dual specificity phosphatases: A gene family for control of MAP kinase function. FASEB J 14: 6-16, 2000.

47. Keyse SM: Protein phosphatases and the regulation of mitogenactivated protein kinase signaling. Curr Opin Cell Biol 12: 186-192, 2000.

48. Chalabi-Dchar M,Cassant-Sourdy S, Duluc C, Fanjul M, Lulka H, Samain R, Roche C, Breibach F, Delisle MB, Poupot M, et al: Loss of somatostatin receptor subtype 2 promotes growth of KRAS-induced pancreatic tumors in mice by activating PI3K signaling and overexpression of CXCL16. Gastroenterology 148: $1452-1465,2015$

49. Chiu FL and Lin JK: Downregulation of androgen receptor expression by luteolin causes inhibition of cell proliferation and induction of apoptosis in human prostate cancer cells and xenografts. Prostate 68: 61-71, 2008.

50. Lim DY, Jeong Y, Tyner AL and Park JH: Induction of cell cycle arrest and apoptosis in HT-29 human colon cancer cells by the dietary compound luteolin. Am J Physiol Gastrointest Liver Physiol 292: G66-G75, 2007.

51. Kim EK and Choi EJ: Pathological roles of MAPK signaling pathways in human diseases. Biochim Biophys Acta 1802 396-405, 2010

52. Wada T and Penninger JM: Mitogen-activated protein kinases in apoptosis regulation. Oncogene 23: 2838-2849, 2004.

53. Chang L and Karin M: Mammalian MAP kinas signaling cascades. Nature 410: 37-40, 2001.

54. McCubrey JA, Steelman LS, Chappell WH, Abrams SL, Wong EW, Chang F, Lehmann B, Terrian DM, Milella M, Tafuri A, et al: Roles of the Raf/MEK/ERK Pathway in cell growth, malignant transformation and drug resistance. Biochim Biophys Acta 1773: 1263-1284, 2007. 
55. Oltersdorf T, Elmore SW, Shoemaker AR, Armstrong RC, Augeri DJ, Belli BA, Bruncko M, Deckwerth TL, Dinges J, Hajduk PJ, et al: An inhibitor of Bcl-2 family proteins induces regression of solid tumours. Nature 435: 677-681, 2005.

56. Huang CY and Tan TH: DUSPs, to MAP kinases and beyond. Cell Biosci 2: 24, 2012.

57. Jeffrey KL, Camps M, Rommel C and Mackay CR: Targeting dual-specificity phosphatases: Manipulating MAP kinase signaling and immune responses. Nat Rev Drug Discov 6: 391-403, 2007

58. Nunes-Xavier C, Romá-Mateo C, Ríos P, Tárrega C, Cejudo-Marín R, Tabernero L and Pulido R: Dual-specificity MAP kinase phosphatases as targets of cancer treatment. Anticancer Agents Med Chem 11: 109-132, 2011.

59. Duronio V: The life of a cell: Apoptosis regulation by the PI3K/PKB pathway. Biochem J 415: 333-344, 2008.

60. Guo C, Yang M, Jing L, Wang J, Yu Y, Li Y, Duan J, Zhou X, Li Y and Sun Z: Amorphous silica nanoparticles trigger vascular endothelial cell injury through apoptosis and autophagy via reactive oxygen species-mediated MAPK/Bcl-2 and PI3K/Akt/mTOR signaling. Int J Nanomedicine 11: 5257-5276, 2016.

61. Gardai SJ, Hildeman DA, Frankel SK, Whitlock BB, Frasch SC, Borregaard N, Marrack P, Bratton DL and Henson PM: Phosphorylation of Bax Ser184 by Akt regulates its activity and apoptosis in neutrophils. J Biol Chem 279: 21085-21095, 2004

62. Yao R and Cooper GM: Requirement for phosphatidylinositol-3 kinase in the prevention of apoptosis by nerve growth factor. Science 267: 2003-2006, 1995.

63. Scheid MP, Lauener RW and Duronio V: Role of phosphatidylinositol 3-OH-kinase activity in the inhibition of apoptosis in haemopoietic cells: Phosphatidylinositol 3-OH-kinase inhibitors reveal a difference in signalling between interleukin-3 and granulocyte-macrophage colony stimulating factor. Biochem J 312: 159-162, 1995.

64. Pande M, Bondy ML, Do KA, Sahin AA, Ying J, Mills GB, Thompson PA and Brewster AM: Association between germline single nucleotide polymorphisms in the PI3K-AKT-mTOR pathway, obesity, and breast cancer disease-free survival. Breast Cancer Res Treat 147: 381-387, 2014.

65. Luster AD: Chemokines-chemotactic cytokines that mediate inflammation. N Engl J Med 338: 436-445, 1998.

66. Struyf S, Proost P and Van Damme J: Regulation of the immune response by the interaction of chemokines and proteases. Adv Immunol 81: 1-44, 2003.
67. Guerreiro R, Santos-Costa Q and Azevedo-Pereira JM: The chemokines and their receptors: Characteristics and physiological functions. Acta Med Port 24 (Suppl 4): S967-S976, 2011 (In Portuguese).

68. Xing YN, Xu XY, Nie XC, Yang X, Yu M, Xu HM, Liu YP, Takano Y and Zheng HC: Role and clinicopath- ologic significance of CXC chemokine ligand 16 and chemokine (C-X-C motif) receptor 6 expression in gastric carcinomas. Hum Pathol 43: 2299-2307, 2012.

69. Wang J, Lu Y, Wang J, Koch AE, Zhang J and Taichman RS: CXCR6 induces prostate cancer progression by the AKT/Mammalian target of rapamycin signaling pathway. Cancer Res 68: 10367-10376, 2008.

70. Chandrasekar B, Bysani S and Mummidi S: CXCL16 signals via Gi, phosphatidylinositol 3-kinase, Akt, I kappa B kinase, and nuclear factor-kappa B and induces cell-cell adhesion and aortic smooth muscle cell proliferation. J Biol Chem 279: 3188-3196, 2004.

71. Deng L, Chen N, Li Y, Zheng H and Lei Q: CXCR6/CXCL16 functions as a regulator in metastasis and progression of cancer. Biochim Biophys Acta 1806: 42-49, 2010.

72. Singh R, Kapur N, Mir H, Singh N, Lillard JW Jr and Singh S: CXCR6-CXCL16 axis promotes prostate cancer by mediating cytoskeleton rearrangement via Ezrin activation and $\alpha v \beta 3$ integrin clustering. Oncotarget 7: 7343-7353, 2016.

73. Hu ZB, Chen Y, Gong YX, Gao M, Zhang Y, Wang GH, Tang RN, Liu H, Liu BC and Ma KL: Activation of the CXCL16/CXCR6 pathway by inflammation contributes to atherosclerosis in patients with End-stage renal disease. Int J Med Sci 13: 858-867, 2016.

74. Hald SM, Kiselev Y, Al-Saad S, Richardsen E, Johannessen C, Eilertsen M, Kilvaer TK, Al-Shibli K, Andersen S, Busund LT, et al: Erratum to: Prognostic impact of CXCL16 and CXCR6 in non-small cell lung cancer: Combined high CXCL16 expression in tumor stroma and cancer cells yields improved survival. BMC Cancer 16: 916, 2016. 